12.1;13.2

\title{
Тест на соответствие режиму холодной полевой эмиссии с применением приближений Елинсона-Шредника и Форбса-Дина (координаты Мерфи-Гуда)
}

\author{
(C) Е.О. Попов, А.Г. Колосько, С.В. Филиппов \\ Физико-технический институт им. А.Ф. Иоффре РАН, Санкт-Петербург, Россия \\ E-mail: e.popov@mail.ioffe.ru
}

Поступило в Редакцию 8 мая 2020 г.

В окончательной редакции 8 мая 2020 г.

Принято к публикации 23 мая 2020 г.

\begin{abstract}
Описан тест, позволяющий определить соответствие экспериментальных данных классической полевой зависимости. Тест представлен в двух вариантах: с применением приближения Елинсона-Шредника к вольт-амперным характеристикам в классических координатах Фаулера-Нордгейма, а также современного приближения Форбса-Дина с модифицированными координатами Мерфи-Гуда. Применение теста показано на примере исследования многоострийного эмиттера на основе углеродных нанотрубок. Представлена методика онлайн-вычисления эффективных параметров эмиттера и проведения теста.
\end{abstract}

Ключевые слова: полевая эмиссия, уравнение Елинсона-Шредника, приближение Форбса-Дина, координаты Мерфи-Гуда, углеродные нанотрубки, онлайн-анализ.

DOI: 10.21883/PJTF.2020.17.49884.18373

Многоострийные полевые эмиттеры являются перспективным элементом для современной вакуумной электроники [1]. Полноценное использование их возможностей требует качественной технологической оптимизации, основанной на изучении фундаментальных закономерностей эмиссионных процессов. Регистрация и анализ вольт-амперных характеристик (BAX) являются основным инструментом для получения информации о полевой эмиссии. Зачастую их сопровождает регистрация картин свечения, возникающих на специальном люминофорном аноде, что позволяет определить расположение эмиссионных центров на поверхности катода и их активность [2]. Сопоставление зарегистрированных BAX с теоретическими представлениями о полевой эмиссии позволяет вычислить важные параметры эмиттера: эффективный коэффициент усиления поля и площадь эмиссии [3], а в некоторых случаях даже работу выхода $\varphi$ [4]. Первыми такими представлениями были результаты исследований Милликена и Лоритсена, которые для построения экспериментальных BAX использовали полулогарифмические координаты $X=1 / U$ и $Y=\lg I$ [5]. Развитая Фаулером и Нордгеймом в 1928 г. теория полевой эмиссии позволила ввести координаты $X=1 / U$ и $Y=\lg \left(I / U^{2}\right)$ более обоснованные теоретически [6]. Исследование отклонения степени $U$ в предэкспоненциальном множителе уравнения от 2 было проведено в работе [7].

Целью настоящей работы является демонстрация экспериментального применения модифицированных координат Фаулера-Нордгейма для быстрой (без введения сложных поправочных функций) обработки ВАХ полевых эмиттеров. В работе проведен тест на соответствие классической полевой эмиссии экспериментальных дан- ных эмиттера на основе углеродных нанотрубок в новых координатах Мерфи-Гуда в режиме реального времени.

В качестве экспериментального образца мы выбрали нанокомпозит углеродные нанотрубки-полистирол (CNT-PS), обладающий перспективными свойствами [8]. Нанокомпозит был нанесен на плоскую металлическую подложку диаметром $1 \mathrm{~cm}$. Анодом являлась такая же подложка, установленная на расстоянии $450 \mu \mathrm{m}$ от катода. Регистрация ВАХ проводилась с помощью комплексной компьютеризированной методики [9]. Уровень вакуума не превышал $10^{-7}$ Torr. Сканирование напряжением производилось в неразрушающем скоростном режиме подачи полусинусоидальных импульсов длительностью $10 \mathrm{~ms}$ (один импульс - одна ВАХ). Регистрация и онлайн-обработка ВАХ осуществлялись с применением специальной программы, написанной на платформе LabView. Обработка BAX заключалась в определении эффективных параметров катода для различных участков ВАX (метод скольжения с заданным диапазоном напряжений, описанный в [10]). Для расчетов использовались два способа построения ВАХ в полулогарифмических координатах.

Классическая теория полевой эмиссии использует приближение плоской атомарно-гладкой проводящей поверхности, обладающей потенциальным барьером на границе эмиттер/вакуум в форме барьера Шоттки с работой выхода $\varphi$ [11]. Формула зависимости плотности тока $J$ от величины электрического поля $F$ на поверхности катода содержит интеграл по энергиям электронов в материале катода. Интеграл этот можно брать численно [12], но чаще всего применяется ряд упрощений для получения уравнения полевой эмиссии с экспоненциальной зависимостью $J(F)$, содержащий 
специальные математические функции $\tau_{\mathrm{F}}$ и $v_{\mathrm{F}}$, взятые на уровне Ферми (режим глубокого туннелирования), либо их табулированные значения [13]. Запишем это уравнение в формулировке безразмерного поля $f$

$$
J=a_{\mathrm{FN}} \tau_{\mathrm{F}}^{-2} \varphi^{-1} F_{R}^{2} f^{2} \exp \left[-v_{\mathrm{F}} b_{\mathrm{FN}} \varphi^{3 / 2} /\left(F_{R} f\right)\right],
$$

где $J-$ плотность тока $\left[\mathrm{A} / \mathrm{m}^{2}\right]$, $a_{\mathrm{FN}}=1.54 \cdot 10^{-6} \mathrm{~A} \cdot \mathrm{eV} \cdot \mathrm{V}^{-2}$ и $b_{\mathrm{FN}}=6.83 \cdot 10^{9} \mathrm{eV}^{-3 / 2} \cdot \mathrm{V} / \mathrm{m}$ первая и вторая константы Фаулера-Нордгейма, $\varphi$ - работа выхода поверхности эмиттера $[\mathrm{eV}]$, $f=F / F_{R}$ - безразмерное поле на поверхности, связанное с полем снятия потенциального барьера $F_{R}=\varphi^{2} c_{S}^{-2}$, где $c_{S}^{2}=1.44 \cdot 10^{-9} \mathrm{eV}^{2} \cdot \mathrm{m} / \mathrm{V}$ - константа Шоттки.

Функции $\tau_{\mathrm{F}}$ и $v_{\mathrm{F}}$ можно заменить значениями вблизи центра диапазона напряжений, допустимых для экспериментально наблюдаемой полевой эмиссии - от $10^{9}$ $\mathrm{V} / \mathrm{m}$ (поле возникновения эмиссии) до $10^{11} \mathrm{~V} / \mathrm{m}$ (поле вероятного термического взрыва катода), например, для $f=0.3$. Одним из самых распространенных является приближение Елинсона-Шредника, которое заменяет $\tau_{\mathrm{F}}^{2}$ на 1.1 и $v_{\mathrm{F}}$ на $v_{\mathrm{F}}=s-u f \approx 0.95-1.03 f[14]$ :

$$
J=\left(a_{\mathrm{FN}} / 1.1\right) \varphi^{-1} F_{R}^{2} f^{2} \exp (1.03 \eta) \exp (-0.95 \eta / f),
$$

где $\eta(\varphi)=b_{\mathrm{FN}} \varphi^{3 / 2} / F_{R}=b_{\mathrm{FN}} c_{S}^{2} \varphi^{-1 / 2}$.

Для перехода от теоретической зависимости $J(f)$ к измеряемым величинам тока $I$ и напряжения $U$ вводится некий характеристический эмиттер, обладающий одинаковым полем на эмиссионной поверхности площадью $A_{e f f}$. Это поле в общем случае связано с приложенным напряжением через размерный коэффициент усиления поля $\alpha_{e f f}: F=\alpha_{e f f} U$. В плоскопараллельной геометрии электродов, когда силовые линии поля параллельны друг другу, а высоты неоднородностей много меньше расстояния между электродами $d_{s e p}$, используется соотношение $\gamma_{e f f}=\alpha_{e f f} d_{\text {sep }}$. В реальности же коэффициент усиления поля определяется формой эмиттера и имеет неравномерное распределение по поверхности [15]. Как было показано в [10], понятие площади эмиссии имеет несколько видов теоретического определения и также зависит от формы эмиттера.

Введение координат Фаулера-Нордгейма $X=1 / U$ и $Y=\ln \left(I / U^{2}\right)$ позволяет „выпрямить“ зависимость $I(U)$. Линейная аппроксимация экспериментальной BAX в этих координатах (назовем ее ВАХ-ФН) позволяет определить значения наклона $S_{f i t}$ и отсечки $I_{f i t} \equiv \ln \left\{R_{f i t}\right\}$. Из уравнения (2) следует взаимосвязь этих величин с параметрами эмиттера

$$
\begin{gathered}
\alpha_{e f f}=-B_{\varphi} / S_{f i t}, \\
A_{e f f}=R_{f i t}\left(S_{f i t}\right)^{2} /\left(A_{\varphi} B_{\varphi}^{2}\right),
\end{gathered}
$$

где $A_{\varphi}=1.4 \varphi^{-1} \exp (10.17 / \sqrt{\varphi})$ и $B_{\varphi}=6.49 \cdot 10^{9} \varphi^{3 / 2}$.

На рис. 1 представлена ВАХ-ФН катода CNT-PS и полученные для нее значения $A_{\text {eff }}$ и $\gamma_{\text {eff }}$, рассчитанные для „полного диапазона“ исследуемых напряжений

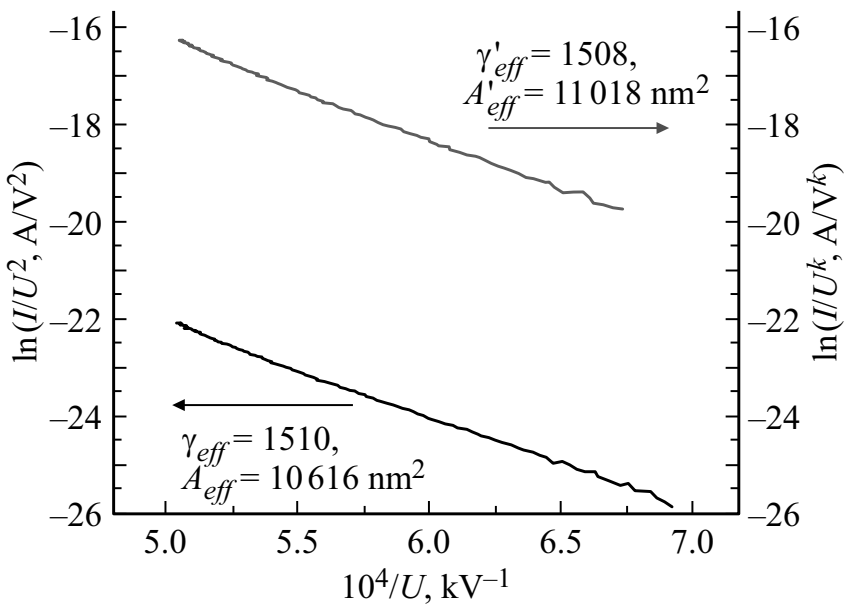

Рис. 1. Экспериментальная BAX, построенная в обычных координатах Фаулера-Нордгейма и модифицированных координатах Мерфи-Гуда.

(1430-1955 V). Во избежание влияния шумов на регрессионный анализ полный диапазон был ограничен снизу уровнем напряжения, начиная с которого среднеквадратичное отклонение данных от линии тренда не превышало 0.002 .

По экспериментальному наклону можно определить не только коэффициент $\alpha_{e f f}$, но и эффективное безразмерное поле на поверхности эмиттера $f$ при заданном напряжении $U$

$$
f=U \alpha_{e f f} / F_{R} \approx-0.95 U \eta / S_{f i t} .
$$

В работе [16] был предложен способ определения (так называемый тест Форбса) того, является ли наблюдаемая в эксперименте эмиссия электронов холодной полевой эмиссией, которую можно описывать семейством уравнений Фаулера-Нордгейма. Тест основан на вычислении диапазона $f_{1} \div f_{2}$ по экспериментальным данным с помощью формулы (5) для минимального и максимального напряжений в эксперименте $U_{\min }$ и $U_{\max }$ с последующим сравнением этого диапазона с табличными полями $f_{\text {low }}$ и $f_{\text {up }}$. Эти допустимые поля были получены в [16] из анализа работ известных ученых, которые регистрировали холодную полевую эмиссию в условиях, близких к идеальным. Значения $f_{\text {low }}$ и $f_{\text {up }}$ определялись возможностью измерения малых токов $(\sim 0.5 \mathrm{nA})$ и устойчивостью эмиттера к большим токам ( $7 \mathrm{~mA} \mathrm{в}$ импульсе). Проведенный Форбсом анализ литературных данных для одноострийных систем показал, что в случае вольфрама $(\varphi=4.5 \mathrm{eV})$ допустимыми полями можно считать диапазон от $f_{\text {low }}=0.15$ до $f_{\text {up }}=0.45$. Смежные диапазоны от $f_{\text {low- }}=0.1$ до $f_{\text {low }}$ и от $f_{\text {up }}$ до $f_{u p-b}=0.75$ можно считать малонадежными, но еще возможными для наблюдения холодной полевой эмиссии. Таким образом, попадание $f_{1}$ и $f_{2}$ в границы $\left[f_{\text {low }} ; f_{\text {up }}\right]$ указывает на соответствие зарегистрированной BAX „ортодоксальной“ теории. Диапазоны для 

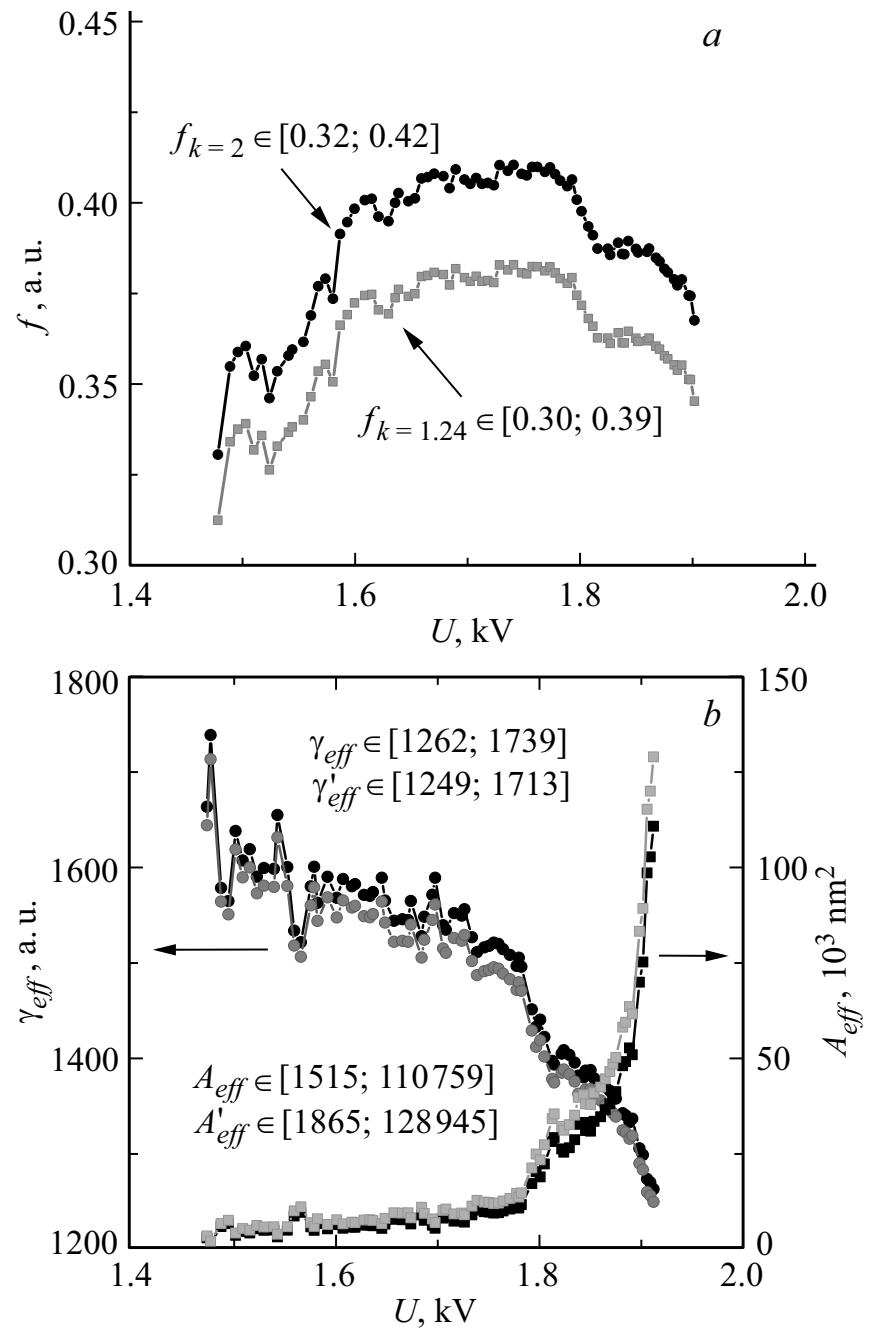

Рис. 2. Изменение эффективных параметров катода при изменении диапазона приложенного напряжения: $a-$ поле на поверхности катода, $b-$ коэффициент усиления поля и площадь эмиссии. Параметры рассчитаны с применением стандартных (черные кривые) и модифицированных (серые кривые) координат Фаулера-Нордгейма. На оси абсцисс отложено среднее значение напряжений выборки в диапазоне $100 \mathrm{~V}$.

эмиттеров с другой работой выхода $\varphi$ рассчитываются аналогичным образом на основании известных предельных токов. Оценка значений $f_{1}$ и $f_{2}$ для полного диапазона напряжений экспериментальных данных (рис. 1) дает значения 0.32 и 0.44 , что близко к верхнему краю допустимого диапазона.

Кроме этой оценки параметров $f_{1}$ и $f_{2}$ по полному диапазону напряжений, а также эффективных значений $A_{e f f}$ и $\gamma_{\text {eff }}$ возможна интервальная оценка, которая учитывает искривление ВАХ-ФН за счет влияния различных эффектов. Такими эффектами могут быть кривизна поверхности катода [10], наличие нескольких групп эмиссионных центров, различающихся коэффициентом усиления поля [17], а также адсорбционные процессы, меняющие работу выхода эмиссионных центров при изменении уровня напряжения [18]. На рис. 2 показано изменение поля $f$, а также значений $A_{\text {eff }}$ и $\gamma_{\text {eff }}$ при изменении приложенного напряжения. Диапазон изменялся методом скольжения по экспериментальным данным небольшим отрезком $100 \mathrm{~V}$. Из построенных зависимостей видно, что с ростом напряжения коэффициент усиления поля монотонно падает, а площадь эмиссии увеличивается (рис. 2,b). Это объясняется растущим относительным вкладом более низких эмиссионных центров, что было показано в работе [17]. При этом локальное поле $f$ демонстрирует перегиб (рис. 2,a), который обусловлен конкурирующими вкладами растущего напряжения и падающего эффективного коэффициента усиления поля.

В приближении Елинсона-Шредника теоретическая ВАХ-ФН прямолинейна. Однако в общем виде зависимости функций $\tau_{\mathrm{F}}$ и $v_{\mathrm{F}}$ от поля делают $\mathrm{BAX}-Ф Н$ немного изогнутой, так что выражения (4), (5) оказываются справедливыми лишь для небольшого участка напряжений. Для выражения этих зависимостей в аналитическом виде применяются различные аппроксимации, которых существует не менее десятка [19]. Наиболее удобным является современный подход, предложенный Форбсом и Дином в работе [20], заменяющий функции $\tau_{\mathrm{F}}$ и $v_{\mathrm{F}}$ приближенными логарифмическими зависимостями:

$$
\begin{gathered}
\tau_{\mathrm{F}}=1+f / 9-(f / 18) \ln f, \\
v_{\mathrm{F}}=1-f+(f / 6) \ln f .
\end{gathered}
$$

Известно, что зависимость $\tau_{\mathrm{F}}$ от поля очень слаба и меняет величину $J$ несущественно, поэтому ее можно приравнять к $\sqrt{1.1}$, так же как в приближении Елинсона-Шредника. Подстановка же зависимости $v_{\mathrm{F}}$ из (7) в общее уравнение (1) дает уравнение, аналогичное (2), но с измененной предэкспоненциальной зависимостью:

$$
J=\left(a_{\mathrm{FN}} / 1.1\right) \varphi^{-1} F_{R}^{2} f^{2-\eta / 6} \exp (\eta) \exp (-\eta / f) .
$$

Анализ экспериментальной ВАХ на основании этого приближения можно провести, используя модифицированные координаты Фаулера-Нордгейма [10,21]: $X^{\prime}=1 / U$ и $Y^{\prime}=\ln \left(I / U^{2-\eta}\right)$. Новые координаты еще не имеют устоявшегося названия. В работе [22] они названы координатами Мерфи-Гуда (MG-plot). Определение соответствующих $S_{f i t}^{\prime}$ и $I_{f i t}^{\prime}$ в этих координатах позволяет оценить основные параметры

$$
\begin{gathered}
\alpha_{e f f}^{\prime}=-B_{\varphi}^{\prime} / S_{f i t}^{\prime}, \\
A_{e f f}^{\prime}=R_{f i t}^{\prime} /\left(A_{\varphi}^{\prime} a^{k}\right),
\end{gathered}
$$

где $A_{\varphi}^{\prime}=1.4 \varphi^{-1} \exp (9.84 / \sqrt{\varphi}) F_{R}^{\eta / 6}$ и $B_{\varphi}^{\prime}=6.83 \cdot 10^{9} \varphi^{3 / 2}$, $k=2-\eta(\sim 1.24$ для $\varphi=4.6 \mathrm{eV})$.

Поле $f$ определяется теперь одинаково для любого диапазона полей по формуле, аналогичной формуле (5):

$$
f=U \alpha_{e f f}^{\prime} / F_{R}=U \eta / S_{f i t}^{\prime} .
$$




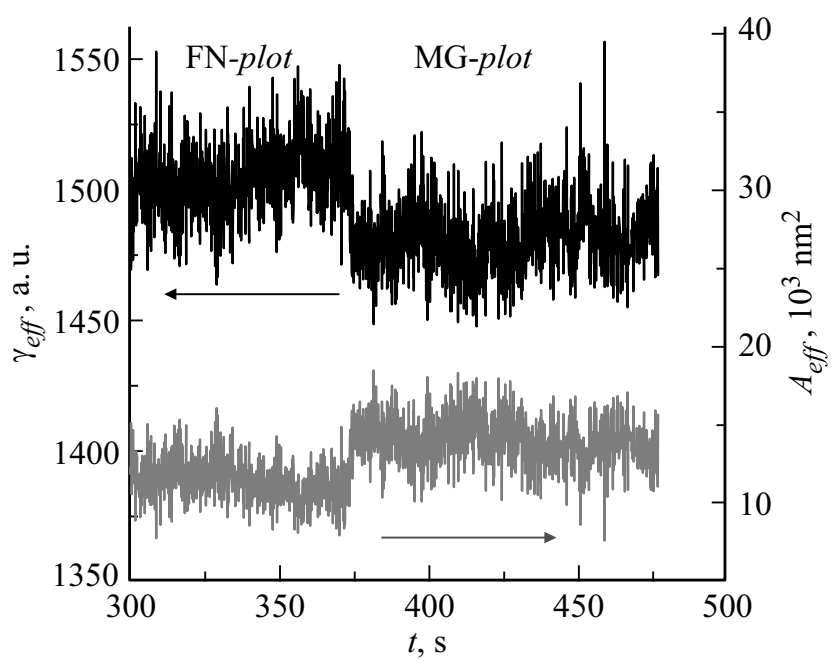

Рис. 3. Непрерывная регистрация параметров катода CNT-PS $A_{\text {eff }}$ и $\gamma_{\text {eff }}$ в режиме онлайн. Резкое изменение уровня значений вызвано переключением измерительной системы на режим онлайн-анализа с модифицированными координатами ВАХ-ФН (координаты Мерфи-Гуда).

На рис. 1 представлена ВАХ-ФН в модифицированных координатах, а также значения $A_{e f f}^{\prime}$ и $\gamma_{e f f}^{\prime}$. Оценка $f_{1}$ и $f_{2}$ дала значения 0.30 и 0.39 , которые находятся дальше от границ запрещенных полей, чем оценка с применением приближения Елинсона-Шредника.

Рис. 3 показывает изменение эффективных параметров катода при переключении измерительной системы на режим анализа ВАХ в модифицированных координатах. Переключение было проведено в процессе регистрации временны́х зависимостей $A_{e f f}$ и $\gamma_{e f f}$ непрерывного потока реализаций $\mathrm{BAX}$ (частота $50 \mathrm{~Hz}$ ) при стабильной работе полевого катода CNT-PS.

Таким образом, мы показали применимость модифицированных координат Фаулера-Нордгейма для анализа свойств многоострийных полевых катодов на примере нанокомпозита CNT-PS. Используемое приближение заменяет функцию $\tau_{\mathrm{F}}$ константой, а $v_{\mathrm{F}}-$ специальной логарифмической аппроксимацией из трех слагаемых. Это позволяет получить теоретически более верные значения эффективных параметров, включая оценку локального поля на поверхности катода. Описан тест Форбса для модифицированных координат, который позволяет проверить соответствие экспериментальных данных режиму холодной полевой эмиссии. Применение новых координат позволяет математически обоснованно учитывать зависимость прозрачности барьера от величины поля независимо от диапазона приложенных напряжений при обработке экспериментальных ВАХ полевых эмиттеров, а также данных трехмерного моделирования распределения полей.

\section{Конфликт интересов}

Авторы заявляют, что у них нет конфликта интересов.

\section{Список литературы}

[1] Егоров Н.В., Шешин Е.П. Автоэлектронная эмиссия. Принципы и приборы. Долгопрудный: Интеллект, 2011. $703 \mathrm{c}$.

[2] Zhao L., Chen Y., Zhang Z., Cao X., Zhang G., She J., Deng S., Xu N., Chen J. // Sci. Rep. 2018. V. 8. P. 12294 $(1-10)$.

[3] Oostrom A.G.J. // Philips Res. Rep. Suppl. 1966. V. 1. P. 1162.

[4] Golubok A.O., Masalov S.A., Tarasov N.A. // Ultramicroscopy. 1992. V. 42-44. P. 1574-1579.

[5] Millikan R.A., Lauritsen C.C. // Proc. Natl. Acad. Sci. USA. 1928. V. 14. P. 45-49.

[6] Nordheim L.W. // Proc. R. Soc. Lond. A. 1928. V. 121. P. 626 639.

[7] Abbott F.R., Henderson J.E. // Phys. Rev. 1939. V. 56. P. 113118.

[8] Sameera I., Bhatia R., Prasad V., Menon R. // J. Appl. Phys. 2012. V. 111. P. 044307 (1-5).

[9] Popov E.O., Kolosko A.G., Filippov S.V., Romanov P.A., Fedichkin I.L. // Mater. Today.: Proc. 2018. V. 5. P. 13800 13806.

[10] Попов Е.О., Колосько А.Г., Чумак М.А., Филиппов С.В. // ЖТФ. 2019. Т. 89. B. 10. C. 1615-1625. DOI: $10.21883 /$ JTF.2019.10.48182.2624 [Пер. версия: 10.1134/S1063784219100177].

[11] Murphy E.L., Good R.H. // Phys. Rev. 1956. V. 102. P. 1464 1473.

[12] Jensen K.L. Introduction to the physics of electron emission. N.J.: John Wiley \& Sons, Inc., 2018. P. 685.

[13] Burgess R.E., Kroemer H., Houston J.M. // Phys. Rev. 1953. V. 90. P. 515.

[14] Елинсон М.И. Ненакаливаемые катоды. М.: Сов. радио, 1974. $336 \mathrm{c}$.

[15] Dall'Agnol F.F., de Assis T.A., Forbes R.G. // J. Phys.: Condens. Matter. 2018. V. 30. P. 375703.

[16] Forbes R.G. // Proc. R. Soc. Lond. A. 2013. V. 469. P. 0271 (1-16).

[17] de Assis T.A., Dall'Agnol F.F., Andrade R.F.S. // J. Phys. D: Appl. Phys. 2016. V. 49. P. 355301 (1-11).

[18] Chen J., Li J., Yang J., Yan X., Tay B.K., Xue Q. // Appl. Phys. Lett. 2011. V. 99. P. 173104.

[19] Forbes R.G., Deane J.H.B. // J. Vac. Sci. Technol. B. 2010. V. 28. P. C2A33-C2A42.

[20] Forbes R.G., Deane J.H.B. // Proc. R. Soc. Lond. A. 2007. V. 463. P. 2907-2927.

[21] Попов Е.О., Колосько А.Г., Филиппов С.В. // Письма в ЖТФ. 2019. Т. 45. B. 18. C. 13-16. DOI: 10.21883/PJTF.2019.18.48230.17898 [Пер. версия: 10.1134/S106378501909027X].

[22] Forbes R.G., Kolosko A.G., Filippov S.V., Popov E.O. // 32nd Int. Vacuum Nanoelectronics Conf. \& 12th Int. Vacuum Electron Sources Conf. Cincinnati, USA, 2019. P. 23. 